\title{
Uniformly finite homology and amenable groups
}

\author{
MATTHIAS BLANK \\ FRANCESCA DIANA
}

\begin{abstract}
Uniformly finite homology is a coarse invariant for metric spaces; in particular, it is a quasi-isometry invariant for finitely generated groups. In this article, we study uniformly finite homology of finitely generated amenable groups and prove that it is infinite-dimensional in many cases. The main idea is to use different transfer maps to distinguish between classes in uniformly finite homology. Furthermore we show that there are infinitely many classes in degree zero that cannot be detected by means.
\end{abstract}

20J05; 43A07

\section{Introduction}

Uniformly finite homology was introduced by Block and Weinberger [5] to study the large-scale structure of metric spaces having bounded geometric complexity. It is a coarse invariant in the sense that two quasi-isometric metric spaces have isomorphic uniformly finite homology. The chains considered are the ones introduced by Roe [19] to define coarse homology, but with an additional boundedness condition on the coefficients (Definition 2.1).

The uniformly finite homology groups of a metric space $X$ are denoted by $H_{*}^{\mathrm{uf}}(X ; \mathbb{R})$. One of the main properties of $H_{0}^{\mathrm{uf}}(X ; \mathbb{R})$ is the relation with the notion of amenability for spaces having coarse bounded geometry (in particular for finitely generated groups). The following result is due to Block and Weinberger [5, Theorem 3.1]:

Theorem 1.1 If $X$ is a metric space with coarse bounded geometry, then $X$ is amenable if and only if $H_{0}^{\mathrm{uf}}(X ; \mathbb{R}) \neq 0$.

On the other hand, it is not so clear how $H_{0}^{\mathrm{uf}}(X ; \mathbb{R})$ looks in the amenable case. Moreover, higher-degree uniformly finite homology groups are not yet well understood; see Block and Weinberger [6] and Dranishnikov [12].

In this paper, we show that uniformly finite homology groups of amenable groups are infinite-dimensional in many cases. Our main result is: 
Theorem 3.8 Let $n \in \mathbb{N}$ and let $G$ be a finitely generated amenable group. Let $H \leq G$ be a subgroup such that $[G: H]=\infty$ and that the map

$$
H_{n}(i): H_{n}(H ; \mathbb{R}) \longrightarrow H_{n}(G ; \mathbb{R})
$$

induced by the inclusion $i: H \longleftrightarrow G$ is nontrivial. Then $\operatorname{dim}_{\mathbb{R}}\left(H_{n}^{\mathrm{uf}}(G ; \mathbb{R})\right)=\infty$.

Therefore, for many amenable groups we can use regular group homology to deduce that uniformly finite homology is infinite-dimensional.

The zero-degree uniformly finite homology group has been used for a number of different applications. For instance, rigidity problems for uniformly discrete metric spaces with bounded geometry have been investigated with the help of uniformly finite homology; see Dymarz [15] and Whyte [23]. Vanishing classes in $H_{0}^{\mathrm{uf}}(X ; \mathbb{R})$ have also been used to construct aperiodic tilings for nonamenable manifolds [5].

Higher-degree uniformly finite homology groups prove to be a powerful tool to investigate large-scale notions of dimensions. Dranishnikov [12; 13; 14] uses the "lifting" map $H_{*}(G ; \mathbb{R}) \rightarrow H_{*}^{\text {uf }}(G ; \mathbb{R})$ to study the macroscopic dimensions of some manifolds. We also present some examples that follow directly from our main theorem. For instance, from the calculation of group homology of nilpotent groups we can deduce:

Example 4.4 (Nilpotent groups) Let $G$ be a finitely generated virtually nilpotent group of Hirsch rank $h \in \mathbb{N}$. Then

$$
H_{k}^{\mathrm{uf}}(G ; \mathbb{R})= \begin{cases}\text { infinite-dimensional } & \text { if } k \in\{0, \ldots, h-1\}, \\ \mathbb{R} & \text { if } k=h, \\ 0 & \text { else. }\end{cases}
$$

Our main technique for proving Theorem 3.8 is to use invariant means on $G$ and the corresponding transfer maps to distinguish between different homology classes. This is straightforward in the degree-zero case and doing so we give a very simple proof that any infinite amenable group $G$ has infinite-dimensional homology group $H_{0}^{\mathrm{uf}}(G ; \mathbb{R})$. In order to extend this to the higher-degree case, we construct functions in $\ell^{\infty}(G)$ that are invariant with respect to a subgroup $H \leq G$ and can be distinguished by means.

We also define a subspace $\hat{H}_{0}^{\text {uf }}(G ; \mathbb{R}) \subset H_{0}^{\text {uf }}(G ; \mathbb{R})$ of classes that can $n o t$ be detected by means and hence are different from the classes constructed before. In the last part of this article, we give a geometric condition for classes in uniformly finite homology to be in $\hat{H}_{0}^{\text {uf }}(G ; \mathbb{R})$. We then present a method of differentiating between such classes, following Whyte. Hence we can show:

Theorem 5.1 Let $G$ be a finitely generated infinite amenable group. Then

$$
\operatorname{dim}_{\mathbb{R}} \hat{H}_{0}^{\mathrm{uf}}(G ; \mathbb{R})=\infty .
$$


The article is structured in the following way. In Section 2 we introduce uniformly finite homology both following Block and Weinberger and in terms of group homology with $\ell^{\infty}$ coefficients and discuss its properties most relevant for this article. Section 3 contains our main results and proofs. In Section 4 we present some examples and immediate applications of our main theorem. Finally, in Section 5 we introduce and study sparse classes in $H_{0}^{\mathrm{uf}}(G ; \mathbb{R})$ and prove Theorem 5.1.

Acknowledgments We thank Cristina Pagliantini for numerous fruitful discussions. We are grateful to Piotr Nowak for coming to Regensburg and suggesting the question. Many thanks to Cristina Pagliantini and Malte Röer for their support. Finally, we especially thank Clara Löh for many helpful suggestions and discussions.

\section{Basic facts and notation}

In this section we fix some notation and present the main object we will investigate, namely uniformly finite homology. This was introduced by Block and Weinberger as a coarse homology invariant. The basic references for uniformly finite homology are [5; 23] and Nowak and $\mathrm{Yu}$ [17]. We will also define group homology with $\ell^{\infty}$ coefficients. For finitely generated groups there is a canonical isomorphism between the corresponding chain complexes (Remark 2.6). Hence, to study uniformly finite homology we will often use the description in terms of $\ell^{\infty}$ coefficients.

Definition 2.1 Let $(X, d)$ be a metric space.

(i) For each $n \in \mathbb{N}$ let $C_{n}^{\text {uf }}(X ; \mathbb{R})$ be the vector space of functions $c: X^{n+1} \rightarrow \mathbb{R}$ satisfying:

(a) The map $c$ is bounded.

(b) For all $r \in \mathbb{R}_{>0}$ there exists a $K_{r} \in \mathbb{R}_{>0}$ (depending on $c$ ) such that for all $y \in X^{n+1}$

$$
\left|\left\{x \in B_{r}(y) \mid c(x) \neq 0\right\}\right| \leq K_{r} .
$$

Here we consider $X^{n+1}$ endowed with the metric

$$
d_{n}(x, y):=\max _{i \in\{0, \ldots, n\}} d\left(x_{i}, y_{i}\right) .
$$

(c) There exists an $R_{c} \in \mathbb{R}_{>0}$ (depending on $c$ ) such that for all $x \in X^{n+1}$ for which $\sup _{i, j \in\{0, \ldots, n\}} d\left(x_{i}, x_{j}\right)>R_{c}$, we have $c(x)=0$.

We will write such a function also as a formal sum $\sum_{x \in X^{n+1}} c(x) \cdot x$. 
(ii) Define for each $n \in \mathbb{N}_{>0}$ a boundary operator

$$
\partial_{n}: C_{n}^{\mathrm{uf}}(X ; \mathbb{R}) \longrightarrow C_{n-1}^{\mathrm{uf}}(X ; \mathbb{R})
$$

by setting for each $x \in X$

$$
\partial_{n}(x)=\sum_{j=0}^{n}(-1)^{j}\left(x_{0}, \ldots, \hat{x}_{j}, \ldots, x_{n}\right)
$$

and extending to $C_{n}^{\mathrm{uf}}(X ; \mathbb{R})$ in the obvious way. In this fashion, we get indeed a chain complex.

(iii) The homology of $\left(C_{n}^{\mathrm{uf}}(X ; \mathbb{R}), \partial_{n}\right)_{n \in \mathbb{N}}$ is called the uniformly finite homology of $X$ and denoted by $H_{*}^{\text {uf }}(X ; \mathbb{R})$.

An important fact about uniformly finite homology is that it is a coarse invariant; see [5, Proposition 2.1].

Proposition 2.2 Let $X$ and $Y$ be metric spaces and $f: X \rightarrow Y$ a quasi-isometry. Then $f$ induces a chain map

$$
\begin{aligned}
C_{n}^{\mathrm{uf}}(X ; \mathbb{R}) & \longrightarrow C_{n}^{\mathrm{uf}}(Y ; \mathbb{R}), \\
\sum_{x \in X^{n+1}} c(x) \cdot x & \longmapsto \sum_{x \in X^{n+1}} c(x) \cdot\left(f\left(x_{0}\right), \ldots, f\left(x_{n}\right)\right) .
\end{aligned}
$$

The induced map in homology

$$
H_{*}^{\mathrm{uf}}(X ; \mathbb{R}) \longrightarrow H_{*}^{\mathrm{uf}}(Y ; \mathbb{R})
$$

is an isomorphism in every degree.

In particular, we can define the uniformly finite homology of a group $G$ with a fixed set of generators $S$, by endowing $G$ with the word metric with respect to $S$. We recall the definition here.

Definition 2.3 Let $G$ be a group with generating set $S$. The word metric on $G$ with respect to $S$ is the metric defined as

$d_{S}(g, h):=\min \left\{n \in \mathbb{N} \mid\right.$ there exist $s_{1}, \ldots, s_{n} \in S \cup S^{-1}$ such that $\left.g^{-1} \cdot h=s_{1} \cdots s_{n}\right\}$ for any $g, h \in G$. 
By Proposition 2.2 the uniformly finite homology of a finitely generated group does not depend on the finite generating set up to canonical isomorphism. Hence, this defines a quasi-isometry invariant for finitely generated groups. Notice that in this case condition (ib) in Definition 2.1 can be dropped.

Most important for us and many applications is the following result of Block and Weinberger [5, Theorem 3.1]:

Theorem 2.4 Let $G$ be a finitely generated group. Then $G$ is amenable if and only if $H_{0}^{\mathrm{uf}}(G ; \mathbb{R}) \neq 0$.

We define now group homology with $\ell^{\infty}$ coefficients. Let $G$ be a (discrete) group. Consider the $\mathbb{R}[G]$-chain complex $\left(C_{*}(G ; \mathbb{R}), \partial_{*}\right)$ defined as follows:

(i) For any $n \in \mathbb{N}$, set

$$
C_{n}(G ; \mathbb{R}):=\bigoplus_{\left(g_{0}, \ldots, g_{n}\right) \in G^{n+1}} \mathbb{R} \cdot\left(g_{0}, \ldots, g_{n}\right)
$$

with the $G$-action given by $g \cdot\left(g_{0}, \ldots, g_{n}\right)=\left(g g_{0}, \ldots, g g_{n}\right)$.

(ii) For any $n \in \mathbb{N}_{>0}$, let $\partial_{n}$ be the boundary map

$$
\begin{aligned}
\partial_{n}: C_{n}(G ; \mathbb{R}) & \longrightarrow C_{n-1}(G ; \mathbb{R}), \\
\left(g_{0}, \ldots, g_{n}\right) & \longmapsto \sum_{j=0}^{n}(-1)^{j}\left(g_{0}, \ldots, \hat{g}_{j}, \ldots, g_{n}\right) .
\end{aligned}
$$

Consider the space of real-valued bounded functions on $G, \ell^{\infty}(G)$; this is a left $\mathbb{R}[G]$-module with respect to the action

$$
\begin{aligned}
G \times \ell^{\infty}(G) & \longrightarrow \ell^{\infty}(G), \\
(g, \varphi) & \longmapsto g \cdot \varphi=\left(g^{\prime} \longmapsto \varphi\left(g^{-1} g^{\prime}\right)\right) .
\end{aligned}
$$

Let $C_{*}\left(G ; \ell^{\infty}(G)\right)$ be the $\mathbb{R}$-chain complex given by

$$
C_{*}\left(G ; \ell^{\infty}(G)\right):=\bar{C}_{*}(G ; \mathbb{R}) \otimes_{\mathbb{R}[G]} \ell^{\infty}(G),
$$

where $\bar{C}_{*}(G ; \mathbb{R})$ denotes the right $\mathbb{R}[G]$-module obtained by $C_{*}(G ; \mathbb{R})$ via the canonical involution $g \mapsto g^{-1}$.

Definition 2.5 For a group $G$ we define the group homology of $G$ with coefficients in $\ell^{\infty}(G)$ by $H_{*}\left(G ; \ell^{\infty}(G)\right):=H_{*}\left(C_{*}\left(G ; \ell^{\infty}(G)\right)\right)$.

The following remark was observed by Brodzki, Niblo and Wright [7]: 
Remark 2.6 For a finitely generated group $G$, the uniformly finite chain complex $\left(C_{*}^{\mathrm{uf}}(G ; \mathbb{R}), \partial_{*}\right)$ is canonically chain isomorphic to $\left(C_{*}\left(G ; \ell^{\infty}(G)\right), \partial_{*}\right)$.

Indeed, for any $n \in \mathbb{N}$, the simplices in a fixed uniformly finite $n$-chain $c$ are tuples $\left(g_{0}, \ldots, g_{n}\right) \in G^{n+1}$ having diameter less than a uniform constant $R_{c} \in \mathbb{R}_{>0}$. These simplices are contained in the $G$-orbits of the finitely many simplices of the form $\left(e, t_{1}, \ldots, t_{n}\right)$ of diameter less than $R_{c}$. Hence, the following chain isomorphism is well defined:

$$
\begin{aligned}
\rho_{n}: C_{n}^{\mathrm{uf}}(G ; \mathbb{R}) & \longrightarrow C_{n}\left(G ; \ell^{\infty}(G)\right), \\
\sum_{\left(g_{0}, \ldots, g_{n}\right) \in G^{n+1}} c\left(g_{0}, \ldots, g_{n}\right) \cdot\left(g_{0}, \ldots, g_{n}\right) & \longmapsto \sum_{\left(t_{1}, \ldots, t_{n}\right) \in G^{n}}\left(e, t_{1}, \ldots, t_{n}\right) \otimes \varphi_{\left(t_{1}, \ldots, t_{n}\right)} .
\end{aligned}
$$

Here for each $\left(t_{1}, \ldots, t_{n}\right) \in G^{n}$ the map $\varphi_{\left(t_{1}, \ldots, t_{n}\right)} \in \ell^{\infty}(G)$ is given by

$$
\varphi_{\left(t_{1}, \ldots, t_{n}\right)}(g)=c\left(g^{-1}, g^{-1} \cdot t_{1}, \ldots, g^{-1} \cdot t_{n}\right) \text {. }
$$

In particular, $H_{*}^{\text {uf }}(G ; \mathbb{R}) \cong H_{*}\left(G ; \ell^{\infty}(G)\right)$. Therefore, certain aspects of uniformly finite homology are accessible through methods of ordinary group homology.

We recall the definition of amenable groups that will be the main class of groups we will study in this article.

Definition 2.7 Let $G$ be a group. A mean is a linear map $m: \ell^{\infty}(G) \rightarrow \mathbb{R}$ satisfying the following properties:

(i) We have $m\left(\chi_{G}\right)=1$ for the characteristic function $\chi_{G} \in \ell^{\infty}(G)$ of the group.

(ii) For any $\varphi \in \ell^{\infty}(G)$ such that $\varphi \geq 0$ (ie, $\varphi(g) \geq 0$ for all $g \in G$ ) we have $m(\varphi) \geq 0$.

A mean $m$ is said to be left $G$-invariant if:

(iii) For any $g \in G$ and any $\varphi \in \ell^{\infty}(G)$, we have $m(g \cdot \varphi)=m(\varphi)$.

Similarly one could define right $G$-invariant means.

Definition 2.8 A group $G$ is amenable if it admits a left $G$-invariant mean.

Remark 2.9 The class of amenable groups contains for example all solvable, all finite and all subexponential groups and is closed under taking extensions, subgroups, quotients, etc; see Ceccherini-Silberstein and Coornaert [10] and Paterson [18]. 
For an amenable group $G$, let $M(G)$ be the set of left $G$-invariant means and let $L M(G)$ be its linear span in $\operatorname{Hom}_{\mathbb{R}}\left(\ell^{\infty}(G), \mathbb{R}\right)$. We have the following result due to Chou [11]:

Theorem 2.10 If $G$ is an infinite amenable group, then $G$ has exactly $2^{2^{|G|}}$ left invariant means, where $|G|$ denotes the cardinality of $G$. Thus $L M(G)$ is infinitedimensional.

\section{Main results}

In this section, we compute uniformly finite homology of finitely generated (discrete) amenable groups in many cases: in Theorem 3.7 we show that in degree zero this is always infinite-dimensional, while in Theorem 3.8 we calculate higher-degree uniformly finite homology.

\subsection{Transfer via means}

Let $G$ be an amenable group. Every left $G$-invariant mean $m \in M(G)$ induces a transfer map $m_{*}: C_{*}\left(G ; \ell^{\infty}(G)\right) \rightarrow C_{*}(G ; \mathbb{R})$, which averages the coefficients. Our next proposition is similar to a result of Attie [2, Proposition 2.15]:

Proposition 3.1 Let $G$ be an amenable group and let $\chi_{G} \in \ell^{\infty}(G)$ be the characteristic function of $G$. Then every mean $m \in M(G)$ induces a transfer map

$$
\begin{aligned}
m_{*}: H_{*}\left(G ; \ell^{\infty}(G)\right) & \longrightarrow H_{*}(G ; \mathbb{R}), \\
{[c \otimes \varphi] } & \longmapsto[m(\varphi) \cdot c],
\end{aligned}
$$

which is a left inverse to the map

$$
\begin{aligned}
i_{*}: H_{*}(G ; \mathbb{R}) & \longrightarrow H_{*}\left(G ; \ell^{\infty}(G)\right), \\
{[c] } & \longmapsto\left[c \otimes \chi_{G}\right],
\end{aligned}
$$

induced by the canonical inclusion $\mathbb{R} \hookrightarrow \ell^{\infty}(G)$ as constant functions.

Proof Consider $\mathbb{R}$ as $G$-module with the trivial action; then every $m \in M(G)$ is a $G$-equivariant map $m: \ell^{\infty}(G) \rightarrow \mathbb{R}$ and thus induces a change-of-coefficients chain map

$$
\begin{aligned}
m_{*}: C_{*}\left(G ; \ell^{\infty}(G)\right) & \longrightarrow C_{*}(G ; \mathbb{R}), \\
c \otimes \varphi & \longmapsto m(\varphi) \cdot c .
\end{aligned}
$$


The inclusion $i: \mathbb{R} \rightarrow \ell^{\infty}(G)$ is also $G$-equivariant and induces a chain map

$$
\begin{aligned}
i_{*}: C_{*}(G ; \mathbb{R}) & \longrightarrow C_{*}\left(G ; \ell^{\infty}(G)\right), \\
c & \longmapsto c \otimes \chi_{G} .
\end{aligned}
$$

Obviously, for every mean $m \in M(G)$ the map $m_{*}$ is a left inverse for $i_{*}$. Applying homology proves the claim.

Remark 3.2 Under the canonical identification between uniformly finite homology and homology with $\ell^{\infty}$ coefficients for finitely generated groups, the maps $m_{*}$ defined in Proposition 3.1 translate (by precomposition with $\rho_{*}$ ) to transfer maps for uniformly finite homology that we denote by $\bar{m}_{*}$.

Hence, for any finitely generated amenable group there is an inclusion

$$
H_{*}(G ; \mathbb{R}) \longleftrightarrow H_{*}^{\mathrm{uf}}(G ; \mathbb{R}) .
$$

Remark 3.3 Given $m \in M(G)$, in degree zero we have $C_{0}\left(G ; \ell^{\infty}(G)\right) \cong \ell^{\infty}(G)$ and the transfer map $m_{0}$ coincides with the mean $m$. Also $C_{0}^{\mathrm{uf}}(G ; \mathbb{R}) \cong \ell^{\infty}(G)$ and $\rho_{0}$ is just the canonical inversion

$$
\begin{aligned}
\ell^{\infty}(G) & \longrightarrow \ell^{\infty}(G), \\
\varphi & \longmapsto\left(g \longmapsto \varphi\left(g^{-1}\right)\right) .
\end{aligned}
$$

Therefore the transfer map $\bar{m}_{0}$ on $C_{0}^{\mathrm{uf}}(G ; \mathbb{R})$ coincides with the corresponding rightinvariant mean.

Definition 3.4 The uniformly finite homological dimension of a group $G$ is defined by

$$
\operatorname{hd}_{\mathrm{uf}} G=\sup \left\{n \in \mathbb{N} \mid H_{n}^{\mathrm{uf}}(G ; \mathbb{R}) \neq 0\right\} \in \mathbb{N} \cup\{\infty\} .
$$

Because uniformly finite homology is invariant under quasi-isometry, we obtain the following fact as an immediate corollary of Proposition 3.1.

Corollary 3.5 The Hirsch rank of a finitely generated virtually nilpotent group $G$ equals $\mathrm{hd}_{\mathrm{uf}}(G)$ and is therefore a quasi-isometry invariant.

Proof We can assume $G$ to be nilpotent; indeed, by definition, the Hirsch rank of a virtually nilpotent group is the Hirsch rank of any nilpotent subgroup $G^{\prime} \leq G$ of finite index, and since $G$ is finitely generated it is quasi-isometric to $G^{\prime}$. Clearly $\operatorname{hd}_{\text {uf }} G \leq \operatorname{hd}_{\mathbb{R}} G$, where hd ${ }_{\mathbb{R}}$ denotes the homological dimension of $G$. 
Conversely, for a finitely generated nilpotent group the homological dimension coincides with the largest integer $n$ for which $H_{n}(G ; \mathbb{R}) \neq 0$ and with its Hirsch rank; see Stammbach [21]. In view of the inclusion $H_{*}(G ; \mathbb{R}) \hookrightarrow H_{*}^{\mathrm{uf}}(G ; \mathbb{R})$ obtained in Proposition 3.1, this integer must be smaller than or equal to $\operatorname{hd}_{\mathrm{uf}} G$.

\subsection{Degree zero}

Definition 3.6 We call the subspace

$$
\widehat{H}_{0}^{\mathrm{uf}}(G ; \mathbb{R}):=\left\{c \in H_{0}^{\mathrm{uf}}(G ; \mathbb{R}) \mid \text { for all } m \in M(G), \bar{m}_{0}(c)=0\right\}
$$

the mean-invisible part of $H_{0}^{\mathrm{uf}}(G ; \mathbb{R})$.

Theorem 3.7 Let $G$ be a finitely generated infinite amenable group. Then

$$
\operatorname{dim}_{\mathbb{R}} H_{0}^{\mathrm{uf}}(G ; \mathbb{R}) / \hat{H}_{0}^{\mathrm{uf}}(G ; \mathbb{R})=\infty .
$$

In particular, $\operatorname{dim}_{\mathbb{R}} H_{0}^{\mathrm{uf}}(G ; \mathbb{R})=\infty$.

Proof By definition we have the canonical inclusion

$$
L M(G) \subseteq \ell^{\infty}(G)^{*}=C_{0}\left(G ; \ell^{\infty}(G)\right)^{*} .
$$

Since this inclusion corresponds to the construction of the chain maps in Proposition 3.1 in degree 0 , this induces a well-defined injection

$$
\begin{aligned}
L M(G) & \longleftrightarrow H_{0}\left(G ; \ell^{\infty}(G)\right)^{*}, \\
m & \longmapsto([g \otimes \varphi] \longmapsto m(\varphi)) .
\end{aligned}
$$

In view of Remarks 2.6 and 3.2, this translates into an inclusion

$$
\begin{aligned}
L M(G) & \longleftrightarrow H_{0}^{\mathrm{uf}}(G ; \mathbb{R})^{*}, \\
m & \longmapsto\left([c] \longmapsto \bar{m}_{0}(c)\right) .
\end{aligned}
$$

By the definition of $\hat{H}_{0}^{\mathrm{uf}}(G ; \mathbb{R})$ this also induces an injection

$$
L M(G) \hookrightarrow\left(H_{0}^{\mathrm{uf}}(G ; \mathbb{R}) / \hat{H}_{0}^{\mathrm{uf}}(G ; \mathbb{R})\right)^{*} .
$$

Hence, by Theorem 2.10 we have $\operatorname{dim}_{\mathbb{R}} H_{0}^{\mathrm{uf}}(G ; \mathbb{R}) / \widehat{H}_{0}^{\mathrm{uf}}(G ; \mathbb{R})=\infty$. 


\subsection{Higher degrees}

Now we consider uniformly finite homology in higher degrees. We construct infinitely many different classes coming from elements in $\ell^{\infty}(G)$ that are invariant with respect to the action of an infinite index subgroup.

Theorem 3.8 Let $n \in \mathbb{N}$ and let $G$ be a finitely generated amenable group. Let $H \leq G$ be a subgroup such that $[G: H]=\infty$ and that the map

$$
H_{n}(i): H_{n}(H ; \mathbb{R}) \longrightarrow H_{n}(G ; \mathbb{R})
$$

induced by the inclusion $i: H \hookrightarrow G$ is nontrivial. Then $\operatorname{dim}_{\mathbb{R}}\left(H_{n}^{\mathrm{uf}}(G ; \mathbb{R})\right)=\infty$.

The idea behind the proof of Theorem 3.8 is to construct a family of infinitely many means on $G$ that can be distinguished by a family of $H$-invariant functions (Theorem 3.11). Following Mitchell [16], in Lemma 3.10 we give a condition for a subset $\Gamma \subset G$ to support a left $G$-invariant mean $m$ such that $m\left(\chi_{\Gamma}\right)=1$. We construct infinitely many such subsets and we separate them using Lemma 3.9.

We will also give an alternative proof of Theorem 3.8 in the case that $H$ is a normal subgroup of $G$.

Lemma 3.9 Let $G$ be an amenable group and $H \leq G$ such that $[G: H]=\infty$. Let $\pi: G \rightarrow H \backslash G$ be the canonical projection. Then for any pair of finite subsets $T, T^{\prime} \subseteq G$ there exists $g \in G$ such that $\pi(T \cdot g) \cap \pi\left(T^{\prime}\right)=\varnothing$.

Proof For each $g \in G$ such that $\pi(T \cdot g) \cap \pi\left(T^{\prime}\right) \neq \varnothing$ there exist $t \in T$ and $t^{\prime} \in T^{\prime}$ such that $\operatorname{tg} \in H t^{\prime}$, hence $g \in t^{-1} H t^{\prime}$. Thus, if $\pi(T \cdot g) \cap \pi\left(T^{\prime}\right) \neq \varnothing$ for all $g \in G$, then $G=T^{-1} H T^{\prime}$. Since $G$ is amenable, there exists a bi- $G$-invariant mean $m$, inducing a finitely additive probability measure $\mu$ on the power set of $G$, given by $\mu(A)=m\left(\chi_{A}\right),[10$, Proposition 4.4.4]. Moreover, since $H$ has infinite index in $G$ it follows that $\mu(H)=0$. Then we have

$$
1=\mu(G)=\mu\left(T^{-1} H T^{\prime}\right) \leq \sum_{t \in T, t^{\prime} \in T^{\prime}} \mu\left(t^{-1} H t^{\prime}\right)=\sum_{t \in T, t^{\prime} \in T^{\prime}} \mu(H)=0,
$$

hence a contradiction.

Lemma 3.10 Let $G$ be a finitely generated amenable group and $\Gamma \leq G$ a subset. Assume that for each finite set $F \subset G$ there exists $g \in G$ such that $F \cdot g \subset \Gamma$. Then there exists a left $G$-invariant mean $m$ on $G$ such that $m\left(\chi_{\Gamma}\right)=1$. 
Proof Let $\left(F_{n}\right)_{n \in \mathbb{N}}$ be a (left) Følner sequence in $G$ (Definition 5.5). Choose for each $n \in \mathbb{N}$ a $g_{n} \in G$, such that $F_{n} \cdot g_{n} \subset \Gamma$. Then $\left(F_{n} \cdot g_{n}\right)_{n \in \mathbb{N}}$ is also a left Følner sequence, since for all $h \in G$ and all $n \in \mathbb{N}$

$$
\frac{\left|h \cdot F_{n} \cdot g_{n} \backslash F_{n} \cdot g_{n}\right|}{\left|F_{n} \cdot g_{n}\right|}=\frac{\left|h \cdot F_{n} \backslash F_{n}\right|}{\left|F_{n}\right|} .
$$

For each $n \in \mathbb{N}$ consider the function

$$
\begin{aligned}
m_{n}: \ell^{\infty}(G) & \longrightarrow \mathbb{R}, \\
x & \longmapsto \frac{1}{\left|F_{n}\right|} \sum_{h \in F_{n}} x\left(h \cdot g_{n}\right) .
\end{aligned}
$$

By [10, Theorem 4.9.2] there is a subsequence of $\left(m_{n}\right)_{n \in \mathbb{N}}$ that converges in the weak- $*$-topology to a left $G$-invariant mean $m$. Since $m_{n}\left(\chi_{\Gamma}\right)=1$ for all $n \in \mathbb{N}$, we also have $m\left(\chi_{\Gamma}\right)=1$.

Theorem 3.11 Let $G$ be a finitely generated amenable group and $H \leq G$ a subgroup such that $[G: H]=\infty$. Then there exists an infinite family $\left(m_{j}\right)_{j \in J}$ of left $G$-invariant means and an infinite family $\left(f_{j}\right)_{j \in J}$ of (left) $H$-invariant functions in $\ell^{\infty}(G)$ such that $m_{k}\left(f_{j}\right)=\delta_{k, j}$ for any $k, j \in J$.

Proof Let $n \in \mathbb{N}$ and let $\pi: G \rightarrow H \backslash G$ be the canonical projection. Consider $G$ equipped with the word metric with respect to a (finite) set of generators. We inductively construct finite sets $A_{l}^{k} \subseteq G$ for all $k \in\{1, \ldots, n\}$ and for all $l \in \mathbb{N}$ such that:

- The family $\left(\pi\left(A_{l}^{k}\right)\right)_{k \in\{1, \ldots, n\}, l \in \mathbb{N}}$ is pairwise disjoint.

- For all $k \in\{1, \ldots, n\}$ and $l \in \mathbb{N}$ there exists $g \in G$ such that $A_{l}^{k}=B_{l}(e) \cdot g$, where $B_{l}(e)$ denotes the ball of radius $l$ centered at the identity element.

Let $A_{1}^{1}:=B_{1}(e)$; assume that the sets have been constructed for all indices smaller than or equal to $(l, k)$ (using lexicographic order). Then:

- If $k<n$, by Lemma 3.9 there exists $g \in G$ such that

$$
\pi\left(\bigcup_{\left(l^{\prime}, k^{\prime}\right) \leq(l, k)} A_{l^{\prime}}^{k^{\prime}}\right) \cap \pi\left(B_{l}(e) \cdot g\right)=\varnothing .
$$

Set $A_{l}^{k+1}:=B_{l}(e) \cdot g$. 
- If $k=n$, by Lemma 3.9 there exists $g \in G$ such that

$$
\pi\left(\bigcup_{\left(l^{\prime}, k^{\prime}\right) \leq(l, k)} A_{l^{\prime}}^{k^{\prime}}\right) \cap \pi\left(B_{l+1}(e) \cdot g\right)=\varnothing .
$$

Set $A_{l+1}^{1}:=B_{l+1}(e) \cdot g$.

Now set for all $k \in\{1, \ldots, n\}$

$$
T^{k}:=\bigcup_{l \in \mathbb{N}} H A_{l}^{k}
$$

Notice that $T^{1}, \ldots, T^{n}$ are pairwise disjoint by construction.

For any finite subset $F \subseteq G$, there exists $r \in \mathbb{N}$ such that $F \subseteq B_{r}(e)$, hence for all $k \in\{1, \ldots, n\}$ there exists $g \in G$ such that $F \cdot g \subseteq B_{r}(e) \cdot g \subseteq T^{k}$. So by Lemma 3.10, for each $k \in\{1, \ldots, n\}$ there exists a left $G$-invariant mean $m_{k}$ such that $m_{k}\left(\chi_{T^{k}}\right)=1$. Moreover, for each $k \in\{1, \ldots, n\}$ and for each $j \in\{1, \ldots, n\} \backslash\{k\}$ we have that $m_{k}\left(\chi_{T^{j}}\right)=0$ since the sets $T^{1}, \ldots, T^{n}$ are pairwise disjoint and $m_{k}(G)=1$. By definition of $T^{k}$, the functions $\chi_{T^{k}}$ are $H$-invariant for any $k \in\{1, \ldots, n\}$.

We have constructed a family of left $G$-invariant means $\left\{m_{k}\right\}_{k \in\{1, \ldots, n\}}$ and a family of bounded $H$-invariant functions $\left\{\chi_{T^{k}}\right\}_{k \in\{1, \ldots, n\}}$ on $G$. We can repeat the same construction for any $n \in \mathbb{N}$, so we can have an arbitrarily large finite family of means and of functions satisfying the theorem. Using a slightly different induction step, it is possible to construct a family of finite sets $A_{l}^{k} \subseteq G$ for all $k \in \mathbb{N}$ and for all $l \in \mathbb{N}$ as above. In this way we are able to construct directly an infinite family of means and of functions satisfying the theorem.

We are now ready to prove our main theorem.

First proof of Theorem 3.8 If $\operatorname{dim}_{\mathbb{R}} H_{n}(G ; \mathbb{R})=\infty$ then by Proposition 3.1 we immediately conclude that $H_{n}^{\text {uf }}(G ; \mathbb{R})$ must be infinite-dimensional.

So we assume $\operatorname{dim}_{\mathbb{R}} H_{n}(G ; \mathbb{R})<\infty$. We prove the statement for $H_{n}\left(G ; \ell^{\infty}(G)\right)$ and in view of Remark 2.6 the theorem will also follow for $H_{n}^{\mathrm{uf}}(G ; \mathbb{R})$.

Let $c \in C_{n}(H ; \mathbb{R})$ be a cycle such that $[i(c)] \in H_{n}(G ; \mathbb{R})$ is nontrivial. Consider the space $\ell^{\infty}(G)^{H}:=\left\{\varphi \in \ell^{\infty}(G) \mid\right.$ for all $\left.h \in H, h \cdot \varphi=\varphi\right\}$. Then

$$
S_{c}:=\left\{i(c) \otimes \varphi \in C_{n}\left(G ; \ell^{\infty}(G)\right) \mid \varphi \in \ell^{\infty}(G)^{H}\right\}
$$

is a subspace of cycles in $C_{n}\left(G ; \ell^{\infty}(G)\right)$. Indeed, for every $\varphi \in \ell^{\infty}(G)^{H}$ one can define the $H$-equivariant map $f_{\varphi}: \mathbb{R} \rightarrow \ell^{\infty}(G), 1 \mapsto \varphi$, which induces a chain map $C_{n}\left(i, f_{\varphi}\right): C_{n}(H ; \mathbb{R}) \rightarrow C_{n}\left(G ; \ell^{\infty}(G)\right)$ that maps $c$ to $i(c) \otimes \varphi$. 
Let $\left(m_{j}\right)_{j \in J}$ and $\left(f_{j}\right)_{j \in J}$ be as in Theorem 3.11; since for all $j \in J$, the map $f_{j}$ is $H$-left invariant, the elements $\left(i(c) \otimes f_{j}\right)_{j \in J} \in C_{n}\left(G ; \ell^{\infty}(G)\right)$ belong to $S_{c}$, so they are all cycles in $C_{n}\left(G ; \ell^{\infty}(G)\right)$. Now consider the family of induced transfer maps $\left(m_{j *}\right)_{j \in J} \in \operatorname{Hom}_{\mathbb{R}}\left(H_{n}\left(G ; \ell^{\infty}(G)\right), H_{n}(G ; \mathbb{R})\right)$. For any $k, j \in J$, by Theorem 3.11 $m_{k *}\left(\left[i(c) \otimes f_{j}\right]\right)=\delta_{i, j} \cdot[i(c)]$, hence the family $\left(m_{j *}\right)_{j \in J}$ is linearly independent in $\operatorname{Hom}_{\mathbb{R}}\left(H_{n}\left(G ; \ell^{\infty}(G)\right), H_{n}(G ; \mathbb{R})\right)$. Thus $H_{n}\left(G ; \ell^{\infty}(G)\right)$ is infinite-dimensional.

Now we give a more direct proof of the main result under the additional assumption that $H$ is normal in $G$.

Second proof of Theorem 3.8 Let $H$ now be normal in $G$. Since $H$ is a subgroup of the amenable group $G$, it is also amenable. Let $m_{0}$ be a left $H$-invariant mean. Consider the transfer map

$$
\begin{aligned}
\tau: \ell^{\infty}(G) & \longrightarrow \ell^{\infty}(G / H), \\
\varphi & \longmapsto\left(g H \longmapsto m_{0}\left(\left.\left(g^{-1} \cdot \varphi\right)\right|_{H}\right)\right) .
\end{aligned}
$$

For any $\varphi \in \ell^{\infty}(G)$, the map $\tau(\varphi)$ is well defined. Indeed, let $g_{1}, g_{2}$ be elements of $G$ such that $g_{1} H=g_{2} H$; then $g_{2}=g_{1} h$ for some $h \in H$, hence

$\tau(\varphi)\left(g_{2} H\right)=m_{0}\left(\left.\left(g_{2}^{-1} \cdot \varphi\right)\right|_{H}\right)=m_{0}\left(\left.\left(h^{-1} g_{1}^{-1} \cdot \varphi\right)\right|_{H}\right)=m_{0}\left(\left.\left(g_{1}^{-1} \cdot \varphi\right)\right|_{H}\right)=\tau(\varphi)\left(g_{1} H\right)$

by the $H$-invariance of $m_{0}$. It is clear that $\tau(\varphi)$ is bounded for any $\varphi \in \ell^{\infty}(G)$.

We also have a map induced by the canonical projection $\pi: G \rightarrow G / H$,

$$
\begin{aligned}
\pi^{*}: \ell^{\infty}(G / H) & \longrightarrow \ell^{\infty}(G), \\
\psi & \longmapsto \psi \circ \pi .
\end{aligned}
$$

It is easy to see that $\operatorname{Im}\left(\pi^{*}\right) \subseteq \ell^{\infty}(G)^{H}$; indeed, for any $\psi \in \ell^{\infty}(G / H)$, any $h \in H$ and any $g \in G$,

$$
h \cdot \pi^{*}(\psi)(g)=\pi^{*}(\psi)\left(h^{-1} g\right)=\psi\left(h^{-1} g H\right)=\psi(g H) .
$$

Notice that the equality $h^{-1} g H=g H$ holds since we have assumed $H$ to be normal in $G$. The composition

$$
\ell^{\infty}(G / H) \stackrel{\pi^{*}}{\longrightarrow} \ell^{\infty}(G) \stackrel{-\left.\tau\right|_{\ell \infty(G)} H}{\longrightarrow} \ell^{\infty}(G / H)
$$

is the identity on $\ell^{\infty}(G / H)$. Indeed, for any $\psi \in \ell^{\infty}(G / H)$ and any class $g H \in G / H$ we have

$$
\left.\tau(\psi \circ \pi)(g H)=\left.m_{0}\left(g^{-1} \cdot(\psi \circ \pi)\right)\right|_{H}\right)=m_{0}(h \mapsto \psi(g H))=\psi(g H),
$$


since the function $h \mapsto \psi(g H)$ is constant. In particular, $\left.\tau\right|_{\ell^{\infty}(G)^{H}}$ is surjective. It is easy to see that $\tau\left(\chi_{G}\right)=1$ and that $\tau(\varphi) \geq 0$ for any $\varphi \geq 0$ in $\ell^{\infty}(G)$. Moreover, $\tau$ is $G$-equivariant: indeed, for any $g, g^{\prime} \in G$ and any $\varphi \in \ell^{\infty}(G)$, we have

$$
\begin{aligned}
\tau\left(g^{\prime} \cdot \varphi\right)(g H)=m_{0}\left(\left.\left(g^{-1} \cdot g^{\prime} \cdot \varphi\right)\right|_{H}\right) & =m_{0}\left(\left.\left(\left(g^{\prime-1} g\right)^{-1} \cdot \varphi\right)\right|_{H}\right) \\
& =\tau(\varphi)\left(g^{\prime-1} g H\right)=g^{\prime} \cdot \tau(\varphi)(g H) .
\end{aligned}
$$

By dualising $\tau$ we get a map

$$
\begin{aligned}
\tau^{*}: \ell^{\infty}(G / H)^{*} & \longrightarrow \ell^{\infty}(G)^{*}, \\
F & \longmapsto F \circ \tau .
\end{aligned}
$$

Since $H$ is a normal subgroup of $G$ with infinite index, $G / H$ is also an infinite amenable group, so restricting $\tau^{*}$ to $\operatorname{LM}(G / H)$ we have a well-defined map

$$
\tau^{*}: L M(G / H) \longrightarrow L M(G) \text {. }
$$

Indeed, let $m \in M(G / H)$ be a mean on $G / H$. Then, since $\tau\left(\chi_{G}\right)=1$ and $\tau(\varphi) \geq 0$ for any $\varphi \geq 0$ in $\ell^{\infty}(G)$, it immediately follows that $\tau^{*}(m)$ satisfies conditions (i) and (ii) of Definition 2.7. Condition (iii) follows by the $G$-equivariance of $\tau$, which implies that $\tau^{*}(m)$ is a left $G$-invariant mean.

Let $\left[S_{c}\right]$ be the space of classes represented by elements of $S_{c}$ as defined in the first proof of Theorem 3.8. Now consider the diagram

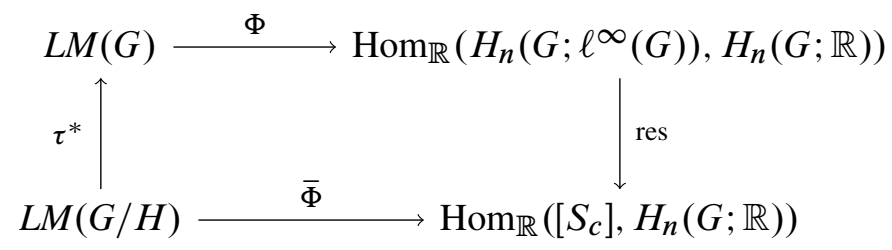

where the upper horizontal map $\Phi$ takes every mean $m \in M(G)$ to the induced homomorphism $m_{*}$. Here res denotes the restriction map and the lower horizontal map is given by composition.

Since $H_{n}(G ; \mathbb{R})$ can be assumed to be finite-dimensional, to prove the theorem it suffices to show that $\bar{\Phi}$ is injective; indeed, by Theorem 2.10 of Chou, since $G / H$ is infinite amenable, $\operatorname{LM}(G / H)$ is infinite-dimensional and from the injectivity of $\bar{\Phi}$ it would follow that $\left[S_{c}\right] \subseteq H_{n}\left(G ; \ell^{\infty}(G)\right)$ must be infinite-dimensional. 
We now show that $\bar{\Phi}$ is injective. So, consider two means $m_{1} \neq m_{2} \in L M(G / H)$; then there exists $\psi \in \ell^{\infty}(G / H)$ such that $m_{1}(\psi) \neq m_{2}(\psi)$. By surjectivity of $\left.\tau\right|_{\ell \infty}(G)^{H}$ we know that there exists $\varphi \in \ell^{\infty}(G)^{H}$ such that $\tau(\varphi)=\psi$. Consider now the image of $[i(c) \otimes \varphi] \in\left[S_{c}\right]$ by the homomorphisms $\bar{\Phi}\left(m_{1}\right), \bar{\Phi}\left(m_{2}\right) \in \operatorname{Hom}\left(\left[S_{c}\right], H_{n}(G ; \mathbb{R})\right)$. We have for $k \in\{1,2\}$

$$
\bar{\Phi}\left(m_{k}\right)([i(c) \otimes \varphi])=\Phi\left(\tau^{*}\left(m_{k}\right)\right)([i(c) \otimes \varphi])=\left[m_{k}(\tau(\varphi)) \cdot i(c)\right]=m_{k}(\psi) \cdot[i(c)] .
$$

Since $m_{1}(\psi) \neq m_{2}(\psi)$ and $[i(c)] \neq 0$, the two classes $m_{1}(\psi) \cdot[i(c)]$ and $m_{2}(\psi) \cdot[i(c)]$ are also different in $H_{n}(G ; \mathbb{R})$, which implies that $\bar{\Phi}\left(m_{1}\right) \neq \bar{\Phi}\left(m_{2}\right)$; in particular, $\bar{\Phi}$ is injective.

\section{Examples}

Corollary 4.1 Let $G$ be a finitely generated amenable group. Assume that $H_{1}(G ; \mathbb{R})$ is nontrivial, ie that the abelianization of $G$ is not a torsion group. Then

$$
H_{1}^{\mathrm{uf}}(G ; \mathbb{R}) \cong \begin{cases}\mathbb{R} & \text { if } G \text { is virtually } \mathbb{Z}, \\ \text { infinite-dimensional } & \text { otherwise. }\end{cases}
$$

Proof Let $g \in G$ be an element such that $(1, g) \in H_{1}(G ; \mathbb{R})$ is a nontrivial cycle. Such a $g$ exists by assumption and the isomorphism $H_{1}(G ; \mathbb{R}) \cong G_{\mathrm{ab}} \otimes \mathbb{R}$. If $G$ is not virtually $\mathbb{Z}$, the claim follows from Theorem 3.8, since then $[G:\langle g\rangle]=\infty$. And for the virtually $\mathbb{Z}$ case see the following example.

Example 4.2 Let $G$ be a finitely generated infinite amenable group. Consider semidirect products of the form $G \rtimes \mathbb{Z}^{l}$ for $l \in \mathbb{N}$. Then for all $k \in\{0, \ldots, l\}$,

$$
\operatorname{dim}_{\mathbb{R}} H_{k}^{\mathrm{uf}}\left(G \rtimes \mathbb{Z}^{l} ; \mathbb{R}\right)=\infty .
$$

Similar results hold if one replaces $\mathbb{Z}^{l}$ by an amenable group with nonvanishing homology in the correct degrees. In particular, for all $l \in \mathbb{N}$

$$
H_{k}^{\text {uf }}\left(\mathbb{Z}^{l} ; \mathbb{R}\right)= \begin{cases}\mathbb{R} & \text { if } k=l, \\ \text { infinite-dimensional } & \text { if } k \in\{0, \ldots, l-1\}, \\ 0 & \text { else. }\end{cases}
$$

Proof The splitting map $\mathbb{Z}^{l} \hookrightarrow G \rtimes \mathbb{Z}^{l}$ induces a nontrivial map in degree $0, \ldots, l$ in homology and hence the first part follows from Theorem 3.8. The product $\mathbb{Z}^{l-1} \times \mathbb{Z}$ is a special case. The degrees $k \geq l$ follow because the group is a Poincare duality group and $H^{0}\left(G ; \ell^{\infty}(G)\right) \cong \ell^{\infty}(G)^{G} \cong \mathbb{R}$ for all groups $G$. 
Example 4.3 For the integral three-dimensional Heisenberg group $\mathrm{Heis}_{3}$ we get

$$
H_{k}^{\mathrm{uf}}\left(\mathrm{Heis}_{3} ; \mathbb{R}\right)= \begin{cases}\mathbb{R} & \text { if } k=3, \\ \text { infinite-dimensional } & \text { if } k \in\{0,1,2\}, \\ 0 & \text { else. }\end{cases}
$$

Proof We only have to consider $k=2$, since the cases $k=0,1$ follow directly from Theorem 3.7 and Corollary 4.1. The higher degrees are a consequence of Poincaré duality. Consider the presentation $\mathrm{Heis}_{3} \cong\left\langle x, y, z \mid[x, y],[y, z], y^{-1}[x, y]\right\rangle$. By Hopf's theorem (see Brown [8, II.5, Theorem 3]), to compute the second homology it suffices to look at the Schur multiplier which, in this case, is generated by the symbols $[x, y]$, $[y, z]$. Hence the inclusion of the subgroup generated by $x$ and $y$ is nontrivial in homology in degree 2 , so we can apply Theorem 3.8 again.

Actually, the last examples are simple special cases of a more general result:

Example 4.4 Let $G$ be a finitely generated virtually nilpotent group of Hirsch rank $h \in \mathbb{N}$. Then

$$
H_{k}^{\mathrm{uf}}(G ; \mathbb{R})= \begin{cases}\mathbb{R} & \text { if } k=h, \\ \text { infinite-dimensional } & \text { if } k \in\{0, \ldots, h-1\}, \\ 0 & \text { else. }\end{cases}
$$

Proof We closely follow the calculation of the homology groups of nilpotent groups of Baumslag, Miller and Short [4] to see that our condition in Theorem 3.8 is satisfied in degrees $0, \ldots, h-1$. After passing to a finite-index subgroup, we may assume $G$ is torsion-free and nilpotent. Then we can write $G$ as a split extension of the form

$$
1 \longrightarrow N \longrightarrow G \longrightarrow \mathbb{Z} \longrightarrow 1
$$

for $N \subset G$ a normal subgroup of Hirsch rank $h-1$. The Hochschild-Serre spectral sequence for this split extension induces a short exact sequence

$$
0 \longrightarrow H_{0}\left(\mathbb{Z} ; H_{i}(N ; \mathbb{R})\right) \longrightarrow H_{i}(G ; \mathbb{R}) \longrightarrow H_{1}\left(\mathbb{Z} ; H_{i-1}(N ; \mathbb{R})\right) \longrightarrow 0
$$

for all $i \in \mathbb{N}_{>0}$. The map on the left-hand side is one edge map of the spectral sequence and is induced by the canonical map $H_{i}(N ; \mathbb{R}) \rightarrow H_{i}(G ; \mathbb{R})$ under the identification $H_{i}(N ; \mathbb{R})_{\mathbb{Z}} \cong H_{0}\left(\mathbb{Z}, H_{i}(N ; \mathbb{R})\right)$; see Weibel [22]. In particular, the canonical map $H_{i}(N ; \mathbb{R}) \rightarrow H_{i}(G ; \mathbb{R})$ is nontrivial if $H_{i}(N ; \mathbb{R})_{\mathbb{Z}}$ is nontrivial. But these homology groups are nontrivial [4, proof of Theorem 16]. The degrees $k \geq h$ follow since finitely generated nilpotent groups are Poincaré duality groups [8, VIII.10, Example 1]. 
Example 4.5 Consider $A \in \operatorname{Sl}(2, \mathbb{Z})$. Then for the semidirect product $\mathbb{Z}^{2} \rtimes_{A} \mathbb{Z}$ given by the action of $\mathbb{Z}$ on $\mathbb{Z}^{2}$ induced by $\mathrm{A}$, we have

$$
H_{k}^{\mathrm{uf}}\left(\mathbb{Z}^{2} \rtimes_{A} \mathbb{Z} ; \mathbb{R}\right)= \begin{cases}\mathbb{R} & \text { if } k=3, \\ \text { infinite-dimensional } & \text { if } k \in\{0,1,2\}, \\ 0 & \text { else. }\end{cases}
$$

In particular, this example includes cocompact lattices in Sol [20].

Proof This is similar to Example 4.4. We only have to consider $k=2$; the other cases follow as in the other examples. By the Hochschild-Serre spectral sequence we get a short exact sequence

$$
0 \longrightarrow H_{0}\left(\mathbb{Z} ; H_{2}\left(\mathbb{Z}^{2} ; \mathbb{R}\right)\right) \longrightarrow H_{2}\left(\mathbb{Z}^{2} \rtimes_{A} \mathbb{Z} ; \mathbb{R}\right) \longrightarrow H_{1}\left(\mathbb{Z} ; H_{1}\left(\mathbb{Z}^{2} ; \mathbb{R}\right)\right) \longrightarrow 0 .
$$

By Poincaré duality and the universal coefficient theorem, the dimension of the middle term is equal to $\operatorname{dim}_{\mathbb{R}} H_{1}\left(\mathbb{Z} \rtimes_{A} \mathbb{Z} ; \mathbb{R}\right)=3$. Since the dimension of the term at the righthand-side is at most 2 , the map $H_{0}\left(\mathbb{Z} ; H_{2}\left(\mathbb{Z}^{2} ; \mathbb{R}\right)\right) \rightarrow H_{2}\left(\mathbb{Z}^{2} \rtimes_{A} \mathbb{Z} ; \mathbb{R}\right)$ is nontrivial. In particular the canonical map $H_{2}\left(\mathbb{Z}^{2} ; \mathbb{R}\right) \rightarrow H_{2}\left(\mathbb{Z}^{2} \rtimes_{A} \mathbb{Z} ; \mathbb{R}\right)$ is also nontrivial. Hence $H_{2}^{\mathrm{uf}}\left(\mathbb{Z}^{2} \rtimes_{A} \mathbb{Z} ; \mathbb{R}\right)$ is infinite-dimensional by Theorem 3.8 .

There are many well-known examples of finitely generated amenable groups having nontrivial real homology in each degree. If $G$ is such a group, the group $G \times \mathbb{Z}$ satisfies $\operatorname{dim}_{\mathbb{R}} H_{n}^{\mathrm{uf}}(G \times \mathbb{Z} ; \mathbb{R})=\infty$ for all $n \in \mathbb{N}$ by Theorem 3.8. For instance:

Example 4.6 There exists a finitely presented metabelian group $G$ such that for all $n \in \mathbb{N}$,

$$
\operatorname{dim}_{\mathbb{R}} H_{n}^{\mathrm{uf}}(G ; \mathbb{R})=\infty
$$

Proof For example, Baumslag and Dyer have shown [3] that Baumslag's metabelian group $B:=\left\langle a, s, t \mid a^{s}=a \cdot a^{t},\left[a, a^{t}\right]=1=[s, t]\right\rangle$ has nontrivial homology in degree $n \geq 3$. Set $G:=B \times \mathbb{Z}^{3}$.

Example 4.7 Consider Thompson's group $F:=\left\langle x_{0}, x_{1}, \ldots\right| x_{n}^{x_{i}}=x_{n+1}$ for $\left.i<n\right\rangle$. We have

$$
F \text { amenable } \Longrightarrow \text { for all } n \in \mathbb{N}, \operatorname{dim}_{\mathbb{R}} H_{n}^{\text {uf }}(F ; \mathbb{R})=\infty
$$

Proof We follow Kenneth Brown's calculation of the homology of $F$ [9]. Consider $F$ as the group of dyadic piecewise linear homeomorphisms $[0,1] \rightarrow[0,1]$. 
Brown observes that in this description the commutator subgroup of $F$ can be seen as $F^{\prime}=\left\{f \in F \mid f^{\prime}(0)=1=f^{\prime}(1)\right\}$. There is a product map

$$
\begin{aligned}
*: F \times F & \longrightarrow F \\
& (f, g) \longmapsto\left(t \longmapsto\left\{\begin{array}{ll}
f(2 \cdot t) / 2 & 0 \leq t \leq \frac{1}{2} \\
g(2 \cdot t-1) / 2 & \frac{1}{2} \leq t \leq 1
\end{array}\right)\right.
\end{aligned}
$$

Define the subgroup $G:=\left\{f \in F \mid f^{\prime}(0)=1\right\} \leq F$. The product map restricts both to $F^{\prime}$ and $G$ and induces a natural product in homology on $H_{*}\left(F^{\prime} ; \mathbb{R}\right), H_{*}(G ; \mathbb{R})$ and $H_{*}(F ; \mathbb{R})$.

Brown [9, Theorem 4.1] shows that $H_{*}(F ; \mathbb{R})$ is generated as a ring by elements $\varepsilon, \alpha, \beta$, that alternating products $\alpha \cdot \beta \cdot \alpha \cdots$ and $\beta \cdot \alpha \cdot \beta \cdots$ form a basis of $H_{*}(F ; \mathbb{R})$ in positive degree and that the image of $H_{*}\left(F^{\prime} ; \mathbb{R}\right) \rightarrow H_{*}(F ; \mathbb{R})$ contains $\alpha \cdot \beta \in H_{2}(F ; \mathbb{R})$ and hence is nontrivial in even degrees.

Since $F^{\prime}$ is strictly contained in $G$, the image of the map $H_{*}(G ; \mathbb{R}) \rightarrow H_{*}(F ; \mathbb{R})$ contains a nontrivial linear combination of $\alpha$ and $\beta$ (since they form a basis of $H_{1}(F ; \mathbb{R})$ ), hence the map $H_{*}(G ; \mathbb{R}) \rightarrow H_{*}(F ; \mathbb{R})$ is nontrivial in each degree and we may apply Theorem 3.8.

\section{Sparse classes in $H_{0}^{\text {uf }}(G ; \mathbb{R})$}

In this section, we give a geometric condition for classes in $H_{0}^{\mathrm{uf}}(G ; \mathbb{R})$ to be meaninvisible and show that in an infinite amenable group, there are infinitely many linear independent classes of this type. In particular, we show the following.

Theorem 5.1 Let $G$ be a finitely generated infinite amenable group. Then

$$
\operatorname{dim}_{\mathbb{R}} \hat{H}_{0}^{\mathrm{uf}}(G ; \mathbb{R})=\infty .
$$

In this section, we always consider finitely generated groups equipped with a word metric with respect to some finite generating set (Definition 2.3).

\subsection{Distinguishing classes by asymptotic behavior}

Let $G$ be a finitely generated discrete group with a word metric $d$. For any $S \subseteq G$ let

$$
\partial_{r}(S):=\{g \in G \mid 0<d(g, S) \leq r\} .
$$

The following theorem due to Whyte [23, Theorem 7.6] gives a characterization of trivial classes in uniformly finite homology in degree 0 . 
Theorem 5.2 Let $G$ be a finitely generated group. Let $c$ be a cycle in $C_{0}^{\mathrm{uf}}(G ; \mathbb{R})$. Then $[c]=0 \in H_{0}^{\mathrm{uf}}(G ; \mathbb{R})$ if and only if there exist $C, r \in \mathbb{N}_{>0}$ such that for all $S \subseteq G$ finite,

$$
\left|\sum_{s \in S} c(s)\right| \leq C \cdot\left|\partial_{r} S\right| .
$$

Remark 5.3 Whyte states Theorem 5.2 for uniformly finite homology with $\mathbb{Z}$ coefficients. However, for infinite finitely generated groups in degree zero, there is an isomorphisms of uniformly finite homology with $\mathbb{Z}$ coefficients and uniformly finite homology with $\mathbb{R}$ coefficients induced by the canonical inclusion $\mathbb{Z} \longleftrightarrow \mathbb{R}[23$, Lemma 7.7]. It is easy to see that this isomorphism translates the original statement of Whyte to Theorem 5.2.

Whyte's criterion can be reformulated in terms of the asymptotic behavior of a degree zero chain in comparison to the behavior of a Følner sequence. First, we introduce a notion to compare the asymptotic behavior of functions:

Definition 5.4 Let $\alpha, \beta: \mathbb{N} \rightarrow \mathbb{R}_{>0}$ be two functions.

(i) We write $\alpha \prec \beta$ if

$$
\lim _{n \rightarrow \infty} \frac{\alpha(n)}{\beta(n)}=0
$$

(ii) We write $\alpha \sim \beta$ if

$$
\lim _{n \rightarrow \infty} \frac{\alpha(n)}{\beta(n)} \in \mathbb{R}_{>0}
$$

(iii) We also write $\alpha \preceq \beta$ if $\alpha \sim \beta$ or $\alpha \prec \beta$.

We recall the definition of a Følner sequence for finitely generated groups. Also recall that a finitely generated group is amenable if and only if it admits a Følner sequence [10, Theorem 4.9.2].

Definition 5.5 Let $G$ be a finitely generated group. A Følner sequence in $G$ is a sequence $\left(S_{j}\right)_{j \in \mathbb{N}}$ of nonempty finite subsets of $G$ such that for each $r \in \mathbb{R}_{>0}$

$$
\lim _{j \rightarrow \infty} \frac{\left|\partial_{r}\left(S_{j}\right)\right|}{\left|S_{j}\right|}=0
$$

Now fix a Følner sequence $S:=\left(S_{j}\right)_{j \in \mathbb{N}}$ of a finitely generated amenable group $G$. 
For all $c \in \ell^{\infty}(G)$ we define a function

$$
\begin{aligned}
\beta_{c}^{S}: \mathbb{N} & \longrightarrow \mathbb{R}, \\
n & \longmapsto \frac{\left|\sum_{s \in S_{n}} c(s)\right|}{\left|S_{n}\right|} .
\end{aligned}
$$

Finally, we also consider the behavior of the boundaries

$$
\begin{aligned}
\sigma_{S}: \mathbb{N} & \longrightarrow \mathbb{R}_{>0}, \\
n & \longmapsto \frac{\left|\partial_{1} S_{n}\right|}{\left|S_{n}\right|} .
\end{aligned}
$$

By comparing the behavior of these functions, we can distinguish between classes in $H_{0}^{\mathrm{uf}}(G ; \mathbb{R})$ :

Lemma 5.6 Let $G$ be a finitely generated amenable group and $S$ a Følner sequence in $G$. Let $c \in \ell^{\infty}(G)$.

(i) We have $0 \preceq \beta_{c}^{S} \preceq 1$.

(ii) If $\beta_{c}^{S} \succ \sigma_{S}$, then $[c] \neq 0 \in H_{0}^{\mathrm{uf}}(G ; \mathbb{R})$.

(iii) Conversely, if $[c] \neq 0 \in H_{0}^{\mathrm{uf}}(G ; \mathbb{R})$ then there exists a Følner sequence $S^{\prime}$ such that $\sigma_{S^{\prime}} \prec \beta_{c}^{S^{\prime}}$.

(iv) More generally, if $\left(c_{n}\right)_{n \in \mathbb{N}}$ is a sequence in $\ell^{\infty}(G)$ such that $\sigma_{S} \prec \beta_{c_{0}}^{S}$ and for all $n \in \mathbb{N}$,

$$
\beta_{c_{n}}^{S} \prec \beta_{c_{n+1}}^{S},
$$

then the family $\left(\left[c_{n}\right]\right)_{n \in \mathbb{N}}$ of classes in $H_{0}^{\mathrm{uf}}(G ; \mathbb{R})$ is linearly independent.

Proof (i) This is obvious by definition.

(ii) Assume $[c]=0$. By Whyte's criterion there exist $C, r \in \mathbb{N}_{>0}$ such that for all $n \in \mathbb{N}$

$$
0<\frac{1}{C} \leq \frac{\left|\partial_{r} S_{n}\right|}{\left|\sum_{s \in S} c(s)\right|} \leq\left|B_{r}(e)\right| \cdot \frac{\left|\partial_{1} S_{n}\right|}{\left|\sum_{s \in S} c(s)\right|} .
$$

Hence $\beta_{c}^{S} \nsucc \sigma_{S}$.

(iii) By Whyte's criterion, if $[c] \neq 0$, for each $n \in \mathbb{N}$ there exists a finite subset $S_{n}^{\prime} \subseteq G$ such that

$$
\left|\sum_{s \in S_{n}^{\prime}} c(s)\right|>n \cdot\left|\partial_{1} S_{n}^{\prime}\right|
$$


hence

$$
\frac{1}{\|c\|_{\infty}} \cdot \frac{\left|\partial_{1} S_{n}^{\prime}\right|}{\left|S_{n}^{\prime}\right|} \leq \frac{\left|\partial_{1} S_{n}^{\prime}\right|}{\left|\sum_{s \in S_{n}^{\prime}} c(s)\right|}<\frac{1}{n} .
$$

In particular $S^{\prime}:=\left(S_{n}^{\prime}\right)_{n \in \mathbb{N}}$ is a Følner sequence and $\sigma_{S^{\prime}} \prec \beta_{c}^{S^{\prime}}$.

(iv) For each $c \in \ell^{\infty}(G)$ define the subspace

$$
C_{0}^{\mathrm{uf}}(G ; \mathbb{R})^{\preceq c}:=\left\{c^{\prime} \in C_{0}^{\mathrm{uf}}(G ; \mathbb{R}) \mid \lim _{n \rightarrow \infty} \frac{\sum_{s \in S_{n}} c^{\prime}(s)}{\sum_{s \in S_{n}} c(s)} \text { exists }\right\} .
$$

Choose a splitting of $\mathbb{R}$-vector spaces $C_{0}^{\mathrm{uf}}(G ; \mathbb{R})=C_{0}^{\mathrm{uf}}(G ; \mathbb{R})^{\preceq c} \oplus V$ and define a linear map

$$
\begin{aligned}
\gamma_{c}^{S}: C_{0}^{\mathrm{uf}}(G ; \mathbb{R})=C_{0}^{\mathrm{uf}}(G ; \mathbb{R})^{\preceq c} \oplus V & \longrightarrow \mathbb{R}, \\
\left(c^{\prime}, c^{\prime \prime}\right) & \longmapsto \lim _{n \rightarrow \infty} \frac{\sum_{s \in S_{n}} c^{\prime}(s)}{\sum_{s \in S_{n}} c(s)} .
\end{aligned}
$$

If $a \in C_{0}^{\mathrm{uf}}(G ; \mathbb{R})$ is a boundary, the function $\beta_{a}^{S} / \sigma_{S}$ is bounded by Whyte's criterion. Therefore, if $\sigma_{S} \prec \beta_{c}^{S}$ then $\beta_{a}^{S} \prec \beta_{c}^{S}$ and hence $\gamma_{c}^{S}(a)=0$. Thus $\gamma_{c}^{S}$ induces a map

$$
\overline{\gamma_{c}^{S}}: H_{0}^{\mathrm{uf}}(G ; \mathbb{R}) \longrightarrow \mathbb{R} \text {. }
$$

In our situation we have $\gamma_{c_{i}}^{S}\left(c_{j}\right)=\delta_{i j}$ for all $j \leq i$ in $\mathbb{N}$. Hence, $\left(\left[c_{n}\right]\right)_{n \in \mathbb{N}}$ is linearly independent.

Example 5.7 The sequence $\left(\left\{n^{k} \mid n \in \mathbb{N}\right\}\right)_{k \in \mathbb{N}_{>0}}$ of subsets of $\mathbb{Z}$ satisfies the conditions of the last part of Lemma 5.6, and hence induces a sequence of linearly independent classes in $H_{0}^{\mathrm{uf}}(\mathbb{Z} ; \mathbb{R})$.

\subsection{Sparse classes}

We now introduce a geometric condition that will ensure that a subset is mean-invisible:

Definition 5.8 Let $G$ be a finitely generated group. We call a subset $\Gamma \subseteq G$ (asymptotically) sparse if there exists $C \in \mathbb{N}$ such that for all $r \in \mathbb{N}_{>0}$, there exists $R \in \mathbb{N}_{>0}$ such that for all $g \in G \backslash B_{R}(e)$,

$$
\left|\Gamma \cap B_{r}(g)\right| \leq C .
$$

Example 5.9 For each $k \in \mathbb{N}_{>1}$ the subsets $\left\{n^{k} \mid n \in \mathbb{N}\right\} \subseteq \mathbb{Z}$ are sparse.

Lemma 5.10 If $\Gamma \subseteq G$ is sparse and $G$ a finitely generated infinite amenable group we have

$$
\left[\chi_{\Gamma}\right] \in \hat{H}_{0}^{\mathrm{uf}}(G ; \mathbb{R}) .
$$


Proof Let $m$ be a left-invariant mean on $G$ and $C \in \mathbb{N}$ as in the definition of sparse. Let $r \in \mathbb{N}_{>0}$. Here we write $\chi(S):=\chi_{S}$ for the characteristic function of any subset $S \subseteq G$. Since $G$ is infinite we have for all $R \in \mathbb{N}_{>0}$ that $\bar{m}(\chi(\Gamma))=\bar{m}\left(\chi\left(\Gamma \backslash B_{R}(e)\right)\right)$. Hence we may assume that for all $g \in G$,

$$
\mid\left\{\gamma \in \Gamma \mid \text { there exists } h \in B_{r}(e) \text { such that } \gamma \cdot h=g\right\}|=| \Gamma \cap B_{r}(g) \mid \leq C .
$$

Therefore the coefficients of $\sum_{h \in B_{r}(e)} \chi(\Gamma \cdot h)$ are bounded by $C$. So we see that

$$
\left|B_{r}(e)\right| \cdot \bar{m}(\chi(\Gamma))=\sum_{h \in B_{r}(e)} \bar{m}(\chi(\Gamma \cdot h))=\bar{m}\left(\sum_{h \in B_{r}(e)} \chi(\Gamma \cdot h)\right) \leq C .
$$

So $\bar{m}(\chi(\Gamma))=0$.

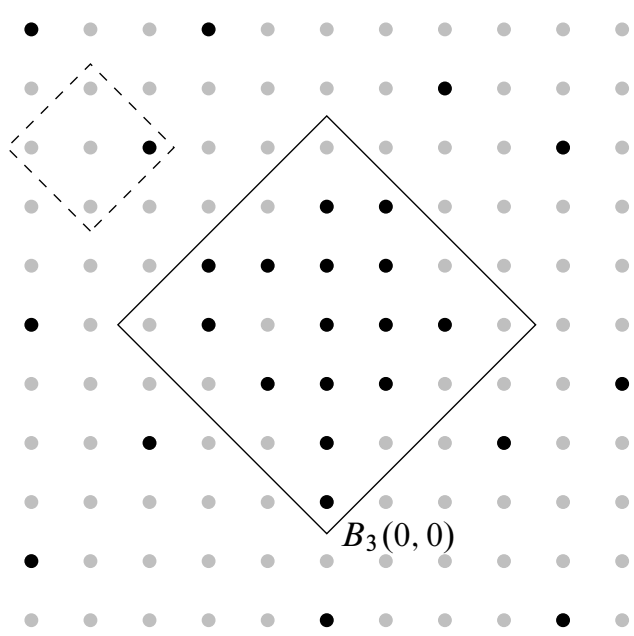

Figure 1: The black dots form a sparse subset in $\mathbb{Z}^{2}$ with the standard word metric

\subsection{Constructing sparse classes}

We recall the notion of tilings, which will be the building blocks for our sparse classes.

Definition 5.11 Let $G$ be a finitely generated group with the word metric. For $r \in \mathbb{N}_{>0}$ we call a subset $T \subseteq G$ an $r$-tiling for $G$ if:

(i) For all $g_{1}, g_{2} \in T$ such that $B_{r}\left(g_{1}\right) \cap B_{r}\left(g_{2}\right) \neq \varnothing$, we have $g_{1}=g_{2}$.

(ii) $G=\bigcup_{g \in T} B_{2 \cdot r}(g)$.

By Zorn's lemma, there exists an $r$-tiling [10, Proposition 5.6.3] for all $r \in \mathbb{N}_{>0}$. 
Lemma 5.12 [10, Proposition 5.6.4] Let $G$ be a finitely generated amenable group and $\left(S_{j}\right)_{j \in \mathbb{N}}$ a Følner sequence. Let $r \in \mathbb{N}_{>0}$ and let $T$ be an $r$-tiling for $G$. Set

$$
T_{j}:=\left\{g \in T \mid B_{r}(g) \subseteq S_{j}\right\} \subseteq S_{j} .
$$

Then there exists an $l(T) \in \mathbb{N}$ such that for all $j \geq l(T)$

$$
\frac{1}{\left|2 \cdot B_{2 \cdot r}(e)\right|} \leq \frac{\left|T_{j}\right|}{\left|S_{j}\right|} \leq \frac{1}{\left|B_{r}(e)\right|} .
$$

Remark 5.13 The idea of the following proof of Theorem 5.1 is the following. Let $\left(S_{j}\right)_{j \in \mathbb{N}}$ be a Følner sequence. For each "ring" $S_{j} \backslash S_{j-1}$ we choose a radius $r(j) \in$ $\mathbb{N}_{>0}$ and put part of an $r(j)$-tiling on this ring; see Figure 2. By letting the sequence $(r(j))_{j \in \mathbb{N}}$ go to infinity, we make sure to get a sparse subset. On the other hand, we let $(r(j))_{j \in \mathbb{N}}$ grow just slowly enough to be sure to get a nontrivial class in $H_{0}^{\mathrm{uf}}(G ; \mathbb{R})$. We then vary the growth of the sequence $(r(j))_{j \in \mathbb{N}}$ and use Lemma 5.6 to get an infinite family of linearly independent classes in $H_{0}^{\mathrm{uf}}(G ; \mathbb{R})$.

Proof of Theorem 5.1 By a result of T Adachi [1] there exists a monotonous and exhausting Følner sequence of $G$. Hence there is a Følner sequence $\left(S_{j}\right)_{j \in \mathbb{N}}$ such that for each $j \in \mathbb{N}$ there exists an $R(j) \in \mathbb{N}_{>0}$ such that

$$
S_{j-1} \subseteq B_{R(j)}(e) \subseteq B_{3 \cdot R(j)}(e) \subseteq S_{j}
$$

Furthermore we can assume $\lim _{j \rightarrow \infty}\left(\left|S_{j-1}\right| /\left|S_{j}\right|\right)=0$.

Choose for each $r \in \mathbb{N}_{>0}$ an $r$-tiling $T^{r}$ of $G$. For each $j \in \mathbb{N}_{>0}$ and each sequence $c=(c(l))_{l \in \mathbb{N}}$ of positive real numbers converging to 0 define $r(j, c)$ to be the maximal number $r \in\{1, \ldots, R(j)\}$ satisfying

$$
\begin{aligned}
\frac{\left|B_{r}(e)\right|}{4 \cdot\left|B_{2 \cdot r}(e)\right|} & \geq \frac{\left|S_{j-1}\right|}{\left|S_{j}\right|}, \\
\frac{1}{4 \cdot\left|B_{2 \cdot r}(e)\right|} & \geq \sqrt{c(j),} \\
l\left(T^{r}\right) & \leq j .
\end{aligned}
$$

If no such $r$ exists, we set $r(j, c)=1$.

The conditions on $r(j, c)$ will force $(r(j, c))_{j \in \mathbb{N}}$ to grow slowly enough so that the class $\Gamma_{c}$ we are about to construct will satisfy $c \prec \beta \underset{\Gamma_{c}}{S}$. 


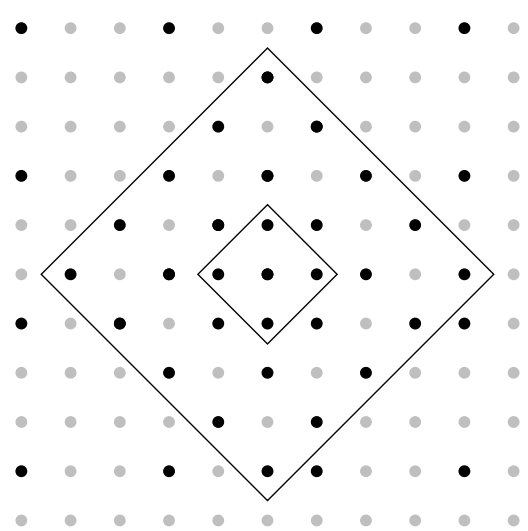

Figure 2: Construction of a sparse subset in $\mathbb{Z}^{2}$ (Remark 5.13)

By our assumption on $\left(S_{j}\right)_{j \in \mathbb{N}}$ we have $\lim _{j \rightarrow \infty} r(j, c)=\infty$. Define the subset

$$
\Gamma_{c}=T_{0}^{r(0, c)} \cup \bigcup_{j \in \mathbb{N}_{>0}}\left(T_{j}^{r(j, c)} \backslash T_{j-1}^{r(j, c)}\right) \subseteq G .
$$

For each $r \in \mathbb{N}$ consider $j \in \mathbb{N}$ such that $r(j, c)>2 \cdot r$. By condition $(*)$ we know that for each $g \in G \backslash S_{j}$ the ball $B_{r}(g)$ intersects at most two sets $S_{l} \backslash S_{l-1}$ and $S_{l+1} \backslash S_{l}$. Because of the tiling condition and since $r(j, c)>2 \cdot r$ the ball also contains at most one point each from

$$
T_{l+1}^{r(l+1, c)} \backslash T_{l}^{r(l+1, c)} \text { and } \quad T_{l}^{r(l, c)} \backslash T_{l-1}^{r(l, c)},
$$

hence $\left|B_{r}(g) \cap \Gamma_{c}\right| \leq 2$, so $\Gamma_{c}$ is sparse. We also have for all $j \in \mathbb{N}_{>0}$, by definition of $\Gamma$,

$$
\begin{aligned}
\frac{\left|\Gamma_{c} \cap S_{j}\right|}{\left|S_{j}\right|} & \geq \frac{\left|T_{j}^{r(j, c)}\right|}{\left|S_{j}\right|}-\frac{\left|T_{j-1}^{r(j, c)}\right|}{\left|S_{j}\right|} \\
& \geq \frac{1}{2 \cdot\left|B_{2 \cdot r(j, c)}(e)\right|}-\frac{\left|S_{j-1}\right|}{\left|B_{r(j, c)}(e)\right| \cdot\left|S_{j}\right|} \\
& \geq \frac{1}{4 \cdot\left|B_{2 \cdot r(j, c)}(e)\right|} \geq \sqrt{c(j)}
\end{aligned}
$$

where the last inequality holds by definition of $r(j, c)$. Therefore, $\beta_{\Gamma_{c}} \succ c$.

Since $\Gamma_{c}$ is sparse, $\lim _{j \rightarrow \infty} \beta_{\Gamma_{c}}(j)=\lim _{j \rightarrow \infty}\left|\Gamma_{c} \cap S_{j}\right| /\left|S_{j}\right|=0$. Now inductively define a sequence $\left(\Gamma_{n}\right)_{n \in \mathbb{N}}$ of sparse subsets by setting $\Gamma_{0}=\Gamma_{\sigma_{S}}$ and $\Gamma_{n+1}=\Gamma_{\beta_{\Gamma_{n}}}$ for all $n \in \mathbb{N}$. Then $\sigma_{S} \prec \beta_{\Gamma_{0}} \prec \beta_{\Gamma_{1}} \prec \cdots$. Hence by Lemma 5.6 we have found a sequence of linearly independent classes in $\hat{H}_{0}^{\mathrm{uf}}(G ; \mathbb{R})$. 


\section{References}

[1] T Adachi, A note on the Folner condition for amenability, Nagoya Math. J. 131 (1993) 67-74 MR1238633

[2] O Attie, Quasi-isometry classification of some manifolds of bounded geometry, Math. Z. 216 (1994) 501-527 MR1288043

[3] G Baumslag, E Dyer, The integral homology of finitely generated metabelian groups, I, Amer. J. Math. 104 (1982) 173-182 MR648485

[4] G Baumslag, C F Miller, III, H Short, Isoperimetric inequalities and the homology of groups, Invent. Math. 113 (1993) 531-560 MR1231836

[5] J Block, S Weinberger, Aperiodic tilings, positive scalar curvature and amenability of spaces, J. Amer. Math. Soc. 5 (1992) 907-918 MR1145337

[6] J Block, S Weinberger, Large scale homology theories and geometry, from: "Geometric topology", (W H Kazez, editor), AMS/IP Stud. Adv. Math. 2, Amer. Math. Soc. (1997) 522-569 MR1470747

[7] J Brodzki, G A Niblo, N Wright, Pairings, duality, amenability and bounded cohomology, J. Eur. Math. Soc. (JEMS) 14 (2012) 1513-1518 MR2966657

[8] KS Brown, Cohomology of groups, Graduate Texts in Math. 87, Springer (1994) MR1324339

[9] KS Brown, The homology of Richard Thompson's group F, from: "Topological and asymptotic aspects of group theory", (R Grigorchuk, M Mihalik, M Sapir, Z Šunik, editors), Contemp. Math. 394, Amer. Math. Soc. (2006) 47-59 MR2216705

[10] T Ceccherini-Silberstein, M Coornaert, Cellular automata and groups, Springer Monographs in Math., Springer (2010) MR2683112

[11] C Chou, The exact cardinality of the set of invariant means on a group, Proc. Amer. Math. Soc. 55 (1976) 103-106 MR0394036

[12] A N Dranishnikov, Macroscopic dimension and essential manifolds, Tr. Mat. Inst. Steklova 273 (2011) 41-53 MR2893542 In Russian; translated in Proc. Steklov Inst. Math. 273 (2011) 35-47

[13] A N Dranishnikov, On macroscopic dimension of rationally essential manifolds, Geom. Topol. 15 (2011) 1107-1124 MR2821571

[14] A N Dranishnikov, On macroscopic dimension of universal coverings of closed manifolds, Trans. Moscow Math. Soc. (2013) 229-244 MR3235798

[15] T Dymarz, Bilipschitz equivalence is not equivalent to quasi-isometric equivalence for finitely generated groups, Duke Math. J. 154 (2010) 509-526 MR2730576

[16] T Mitchell, Constant functions and left invariant means on semigroups, Trans. Amer. Math. Soc. 119 (1965) 244-261 MR0193523 
[17] P W Nowak, G Yu, Large scale geometry, Eur. Math. Soc., Zürich (2012) MR2986138

[18] A L T Paterson, Amenability, Math. Surveys and Monographs 29, Amer. Math. Soc. (1988) MR961261

[19] J Roe, Lectures on coarse geometry, Univ. Lecture Series 31, Amer. Math. Soc. (2003) MR2007488

[20] P Scott, The geometries of 3-manifolds, Bull. London Math. Soc. 15 (1983) 401-487 MR705527

[21] U Stammbach, On the weak homological dimension of the group algebra of solvable groups, J. London Math. Soc. 2 (1970) 567-570 MR0263927

[22] C A Weibel, An introduction to homological algebra, Cambridge Studies in Advanced Math. 38, Cambridge Univ. Press (1994) MR1269324

[23] K Whyte, Amenability, bilipschitz equivalence, and the von Neumann conjecture, Duke Math. J. 99 (1999) 93-112 MR1700742

Fakultät für Mathematik, Universität Regensburg

93040 Regensburg, Germany

matthias.blank@mathematik.uni-regensburg.de, francesca.diana@mathematik. uni-regensburg.de

http://www.mathematik.uni-r.de/blank/,

http://homepages-nw. uni-regensburg.de/ dif13273/

Received: 5 June 2014 